\title{
A Novel Architecture of Radix-3 Singlepath Delay Feedback (R3SDF) FFT Using MCSLA
}

\author{
K. Periyarselvam ${ }^{1}$, G. Saravanakumar ${ }^{2}$, M. Anand ${ }^{3}$ \\ ${ }^{1}$ St Peter's University, India \\ ${ }^{2}$ Department of Electronics and Communication Engineering, VelTech High Tech Dr.Rangarajan Dr.Sagunthala \\ Engineering College, Avadi \\ ${ }^{3}$ Educational and Research Institute University, Chennai-95
}

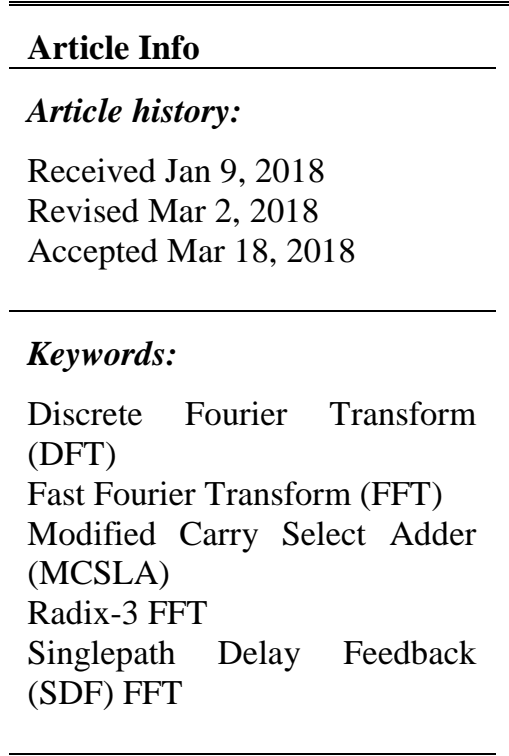

\begin{abstract}
Fast Fourier transform (FFT) is widely used in digital signal processing and telecommunications, particularly in orthogonal frequency division multiplexing systems, to overcome the problems associated with orthogonal subcarriers. A new algorithm of radix-3 FFT has been introduced in this work. The DFT of length $\mathrm{N}$ can be realized from three DFT sequences; each of length N/3.Radix-3 algorithm reduces the number of multiplications required for realizing DFT.A novel design of Radix-3pipelined Single path Delay Feedback (R3SDF) FFT using MCSLA has been proposed in this paper. First, the pipelined radix-3 SDF FFT method has been designed. It has less area and large power consumption and delay. In order to overcome these problems, modified carry select adder structure is used to perform the adder operation for reducing the power consumption and delay. Finally, the MCSLA is integrated into radix-3 SDF FFT processor. The hardware complexity and execution time for implementing radix-3 FFT algorithm can be reduced than other FFTs.
\end{abstract}

Copyright $(2018$ Institute of Advanced Engineering and Science. All rights reserved.

\section{Corresponding Author:}

K. Periyarselvam,

St Peter's University,

India.

Email: periyarvlsi@gmail.com

\section{INTRODUCTION}

Discrete Fourier transform (DFT) is crucial in recent telecommunications and digital signal processing, though this method tends to be computationally rigorous. To conquer this problem, Cooley and Tukey developed the fast Fourier transform (FFT), which has verified predominantly expensive for applications involving orthogonal frequency division multiplexing (OFDM), such as Worldwide Interoperability for Microwave Access (WiMAX), long-term evolution (LTE), asymmetric digital subscriber line (DSL), very-high-speed DSL, and digital audio/video broadcasting (DAB/DVB) systems.

To reduce power consumption and hardware costs, different types of FFT processors has been developed. The memory-based architecture gives a low-power result, though this method suffers from long delay and may need extra buffer space for system synchronization. The pipelined Single-path Delay Feedback (SDF) FFT architecture has been developed to reduce the memory mandatory for memory-based architectures. This approach includes $\mathrm{N}-1$ delay elements, in which the multiplication accounts for less than $50 \%$ of the computation and the control unit design is relatively straightforward. These features are specific advantageous in high-performance designs involving portable digital signal processing devices.

Fixed radix FFT's such as radix 3 FFT are considered to be competitively proficient to radix 2 FFT. In this paper, a new algorithm of pipelined radix-3 SDF FFT using MCSLA has been designed to reduce the number of multiplications. In this radix-3 FFT, the modified carry select adder has been used to perform 
addition operation to reduce the power consumption and also to improve the performance of the FFT processor.

\section{BACKGROUND}

A novel algorithm for execution of radix 3, 6, and 12 FFT has been explained in [1]. The FFT algorithm is evaluated in an ordinary $(1, \mathrm{j})$ complex plane and the number of additions can be extensively reduced, the number of multiplication is also reduced. A well-organized approach to calculate Discrete Fourier Transform (DFT) using Radix-3 algorithm, which is a Fast Fourier Transform (FFT), has been described in [2]. Compared to existing one, it has less multiplication. The matrix created by various powers of twiddle factor is disintegrated into two matrices and it has been revealed that it takes the complex multiplications are less to calculate the result than unique Cooley-Tukey algorithm.

The hardware implementation of mixed radix FFTs with cores of radix 5 and radix 3 as well as the standard radix 2 core has been presented in [3]. The mixed radix FFT is more costly than the radix 2 implementation.A mixed radix FFT of 1200 points need 36 real multipliers in the implementation of pipelined FFT whereas a 2048 radix 2 FFT needs 30 real multipliers. A radix-3 FFT has been described in [4] which the element three-point DFT's needs no multiplications. This results in a reduction in the number of multiplications but a concurrent increase in the number of additions. The algorithm will show an advantage of processors which require more time for multiplication than addition.

A novel FFT algorithm has been developed in [5] together with the design of pipelined architecture. The proposed algorithm has been used to reduce the number of complex multipliers in addition to the size of twiddle factor ROMs. It is proved to be appropriate for large size of FFT VLSI implementation. These FFT architectures are designed for OFDM applications in [6]-[7]. A novel architecture for efficient method of Fast Fourier Transform (FFT) processor [8] to gather the necessities of high speed wireless communication system standards. This paper develops an optimal constant multiplication arithmetic design to multiply a fixed point input by means of one of the numerous current twiddle factor constants.

\section{RADIX-3 FFT ALGORITHM}

Radix-3 FFT algorithm is used to compute Discrete Fourier Transform (DFT).It takes less multiplication than the normal one.Radix-3 FFT algorithm is mainly based on divide and conquer method. It decomposes an N-point DFT into sequentially smaller DFTs.As soon as the number of data points is power of 3 (i.e., $3 n$ ).

The radix-3 algorithm for realization of DFT of length $\mathrm{N}=3 \mathrm{n}(\mathrm{n}=1,2,3, \ldots)$. The DFT of length $\mathrm{N}$ can be realized from three DFT sequences, each of length N/3.If the input signal has length $\mathrm{N}$, direct calculation of DFT needs $\mathrm{O}(\mathrm{N} 2)$ complex multiplications.Radix-3 algorithm which is utilized to reduce the multiplications. The processing time and hardware complexity for implementing radix -3 DFT algorithm can be reduced.

The DFT of $\mathrm{N}$ points is given by

$$
Z(k)=\sum_{n=0}^{N-1} x(n) e^{-j 2 \pi k n / N}, \text { for } \mathrm{k}=0,1,2, \ldots, \mathrm{N}-1
$$

Where $\mathrm{j}=\sqrt{ }-1$

$\mathrm{Z}(\mathrm{k})=$ Transformed Data

$$
\text { Substitute } W_{N}^{k n}=e^{\frac{-j 2 \pi k n}{N}} \text { in }
$$

$$
Z(k)=\sum_{n=0}^{N-1} x(n) W_{N}^{k n}
$$

$W_{N}^{k n}$ is known as twiddle factor. 
The output components are $\mathrm{Z}(0), \mathrm{Z}(1), \mathrm{Z}(2), \ldots, \mathrm{Z}(\mathrm{N}-1)$ are arranged in three groups namely $\mathrm{Z}(3 \mathrm{r}), \mathrm{Z}(3 \mathrm{r}+1)$ and $\mathrm{Z}(\mathrm{N}-3 \mathrm{r}-1)$, where $\mathrm{r}=0,1,2, \ldots, \mathrm{N} / 3-1$.

The following expressions can be derived from equations (2),

$$
Z(3 r)=\sum_{n=0}^{\frac{N}{3}-1}\left[x(n)+x\left(n+\frac{N}{3}\right)+\left(n+\frac{2 N}{3}\right)\right] W_{N}^{k n}
$$

Where $\mathrm{k}=3 \mathrm{r}$ and $\mathrm{r}=0,1,2, \ldots, \mathrm{N} / 3-1$.

$$
Z(3 r+1)=\sum_{n=0}^{N}-1\left[x(n)+W_{N}^{k}(N / 3) x\left(n+\frac{N}{3}\right)+W_{N}^{k(2 N / 3)}\left(n+\frac{2 N}{3}\right)\right] W_{N}^{k n}
$$

Where $\mathrm{k}=3 \mathrm{r}+1$ and $\mathrm{r}=0,1,2, \ldots, \mathrm{N} / 3-1$.

$$
Z(N-3 r-1)=\sum_{n=0}^{\frac{N}{3}-1}\left[x(n)+W_{N}^{k(N / 3)} x\left(n+\frac{N}{3}\right)+W_{N}^{k(2 N / 3)}\left(n+\frac{2 N}{3}\right)\right] W_{N}^{k n}
$$

Where $k=(\mathrm{N}-3 \mathrm{r}-1)$ and $\mathrm{r}=0,1,2 \ldots \mathrm{N} / 3-1$.

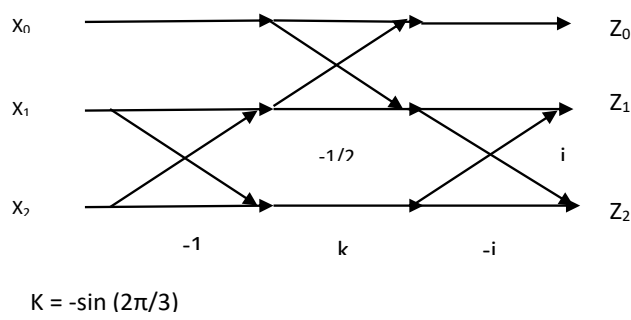

Figure 1. Flow Graph of Radix-3 FFT

\section{PROPOSED PIPELINED STRUCTURE OF RADIX-3 SDF FFT USING MCSLA}

In this paper, a new algorithm of pipelined structure based Radix-3 SDF FFT has been designed for improving the speed. Radix-3 FFT, which is used to reduce the number of multiplication. Single path Delay Feedback FFT is a pipelined based frequency transformation technique. In SDF FFT, the inputs are given in serial manner. The SDF FFT provides high speed operation. This FFT structure consumes more delay and power consumption [9] due to utilizing or storing bulk of unwanted intermediate processing signals. SDF FFT structures have the most proficient memory utilization for pipelined FFT processors.

Figure 2 shows that the architecture of Radix-3 pipelined SDF FFT. This architecture consists of Processing Element (PE), Delay and Twiddle Factor values. Addition and subtraction operation has been performed in the processing element. Initially, the input data of real and imaginary values are given to the first stage. Then the input values are delayed by 1 . The delayed values are given to the processing element.

In the processing element, the addition and subtraction operations are done. After that the values are multiplied by twiddle factor values. Finally, the first stage of output values is controlled by using multiplexor. The first stage output is fed back to the input of second stage. Similarly, the first stage operation has been done in the second stage and third stage. The main disadvantage of single path delay feedback FFT is large power consumption. To overcome this problem, modified carry select adder [10] has been integrated into Radix-3 SDF FFT to perform the efficient adder operation for reducing the power consumption. Modified the full adder structure in the normal CSLA [11] by reducing the number of gates, it is called as Modified Carry Select Adder. 


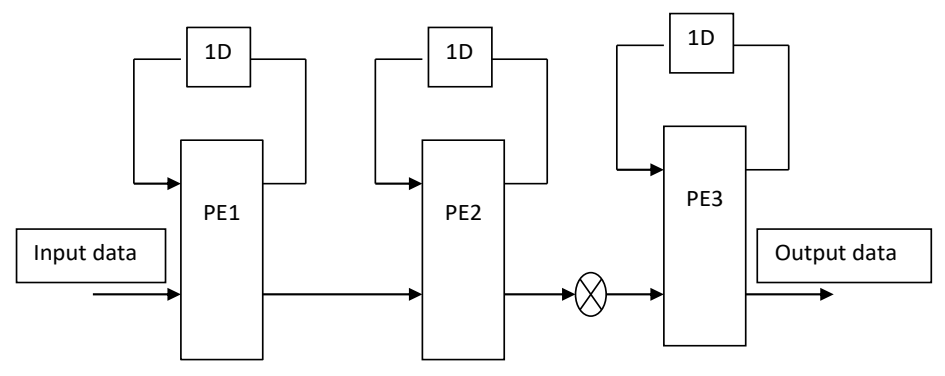

Figure 2. Architecture of Radix-3 Pipelined SDF FFT

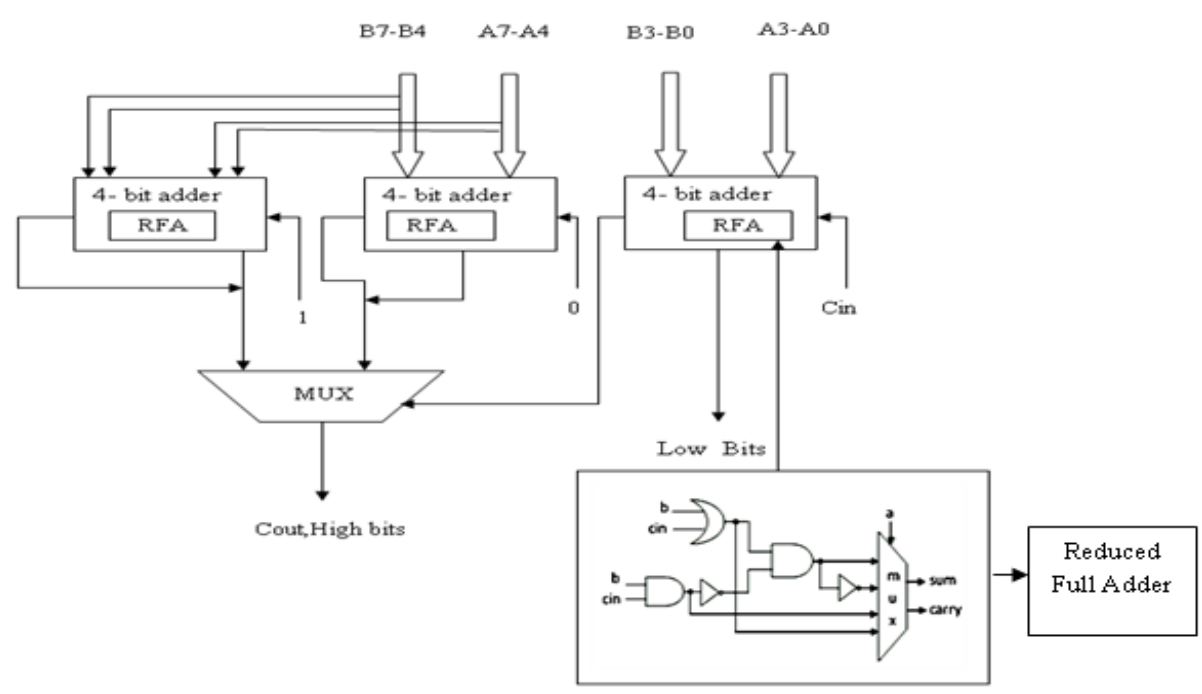

Figure 3. Structure of modified carry select adder

For performing the operation of 3-bit addition, Full Adder circuit consists of 2 XOR gate, 2 AND gate and 1 OR gate. The Full Adder gate count value is 13. RFA circuit [7] has been designed by using minimum number of logic gates. It consists of 2 AND gate, 1 OR gate, 2 NOT gate and 1 multiplexer. The RFA gate count value is 9.Multiplexer (MUX) based Reduced Full Adder circuit has been designed in this paper for improving the performance of digital adder circuits. The structure of modified CSLA is shown in Figure 3.

In the modified CSLA [7] has been used for reducing the power consumption and improving the performance of FFT processor. Compared to regular CSLA, the modified CSLA [7] gives better performance. Finally, the modified CSLA has been integrated into pipelined radix-3 SDF FFT.

\section{RESULTS AND DISCUSSION}

By using Verilog Hardware Description Language (Verilog HDL), the Radix-3 Single-path Delay Feedback (R3SDF) FFT using Modified Carry Select Adder (MCSLA) has been developed. The simulation and synthesis results have been evaluated and estimated by using ModelSim 6.3c and Xilinx 10.1i design tool. The simulation result of proposed Radix-3 pipelined SDF FFT using MCSLA is shown in Figure 4. Comparison analysis of Radix-3 SDF FFT and Radix-3 SDF FFT using MCSLA is shown in Figure 5. 


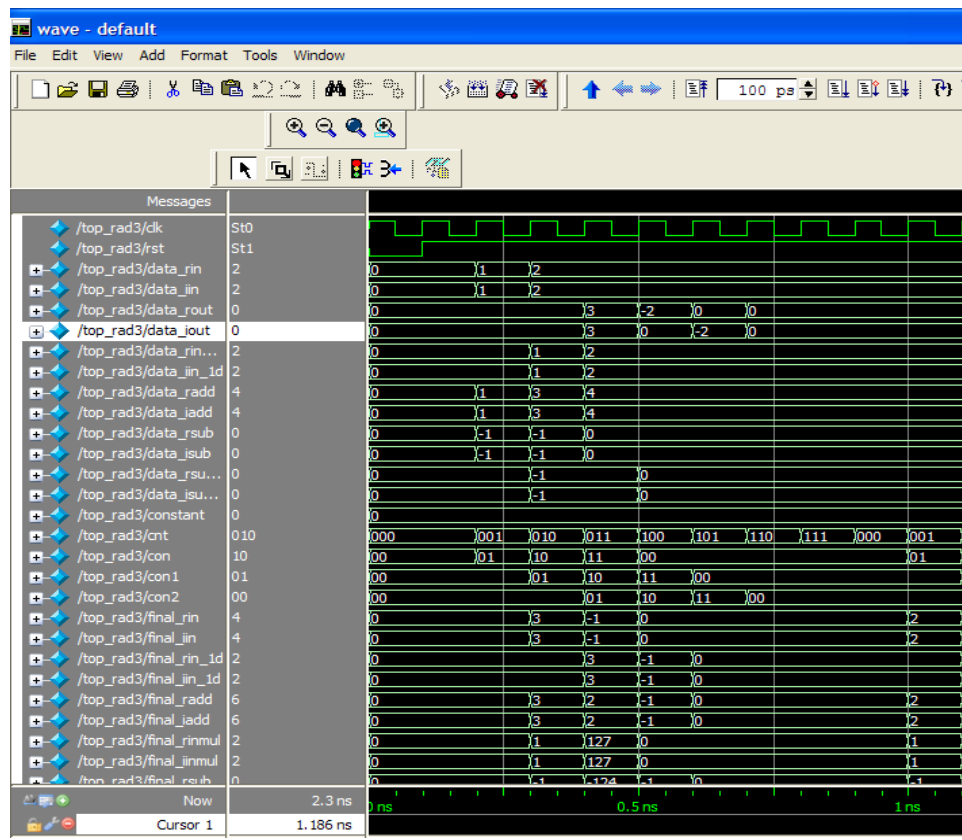

Figure 4. Simulation Result of Proposed Pipelined Radix-3 SDF FFT using Modified Carry Select Adder

Table 1. Comparison Analysis of Pipelined R3SDF FFT and Pipelined R3SDF FFT using Modified Carry Select Adder

\begin{tabular}{ccccc}
\hline Types & Slices & LUTs & Delay (ns) & Power Consumption (mW) \\
\hline $\begin{array}{c}\text { Pipelined Radix-3 SDF FFT } \\
\text { Pipelined Radix-3 SDF FFT using Modified }\end{array}$ & 170 & 318 & 15.173 & 475 \\
$\begin{array}{c}\text { Carry Select Adder } \\
\text { \% Reduction }\end{array}$ & 100 & 180 & 10.507 & 118 \\
& 41.17 & 43.39 & 30.75 & 75.15 \\
\hline
\end{tabular}

Table 1 shows that the number of slices is 170 and100, the number of LUTs is 318 and180, the delay is $15.173 \mathrm{~ns}$ and $10.507 \mathrm{~ns}$ and the power consumption is $475 \mathrm{~mW}$ and $118 \mathrm{~mW}$ in pipelined R3SDF FFT and R3SDF FFT using Modified Carry Select Adder.

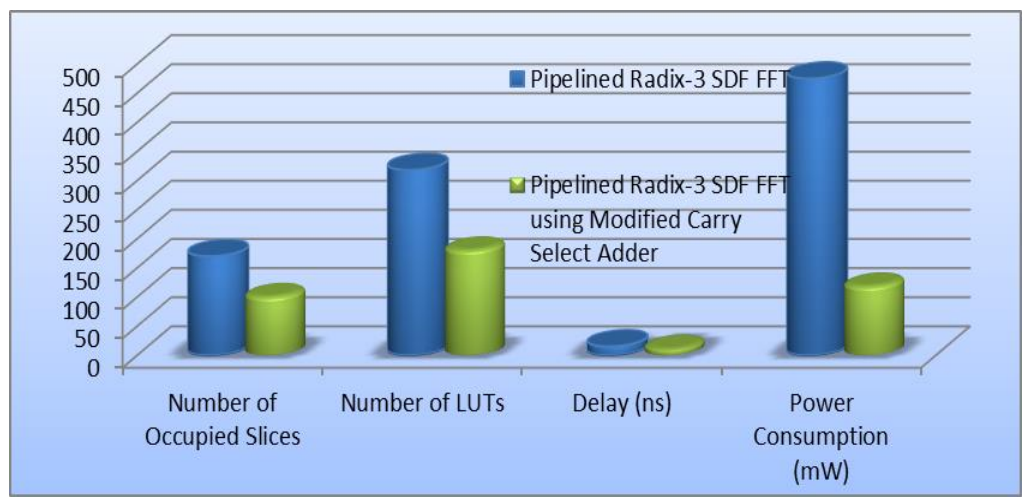

Figure 5. Performance Evaluation of pipelined Radix-3 SDF FFT and Radix-3 SDF FFT using MCSLA

\section{CONCLUSION}

In this paper, a novelty design of pipelined Radix-3 Singlepath Delay Feedback (R3SDF) FFT using modified CSLA has been proposed. The proposed radix-3 FFT has less number of multiplications compared to other FFTs.This algorithm is competitive in speed with radix 2 FFT. In pipelined Radix-3 SDF FFT, the area has been reduced but the delay and power consumption has been increased. In order to overcome this 
problem, modified carry select adder has been used to perform adder operation in Radix-3 SDF FFT. To reduce the area, latency and power consumption is the main motive of this paper. The proposed method offers $41.17 \%$ lessening in occupied slices, $43.39 \%$ decrease in LUTs, $30.75 \%$ reduction in delay and $75.15 \%$ lessening in power consumption than the Radix-3 SDF FFT.

\section{REFERENCES}

[1] Suzuki Y, Sone T, and Kido k. A new FFT algorithm of Radix-3, 6 and 12.IEEE Transactions on acoustics, speech and signal processing. 1986; 34(2):381-383.

[2] Bashar S K. An efficient approach to the computation of fast fourier transform (FFT) by Radix-3 algorithm. IEEE International Conference on Informatics, Electronics \& Vision (ICIEV).2013; 1-5.

[3] Lofgren J, and Nilsson P. On hardware implementation of radix 3 and radix 5 FFT kernels for LTE systems. IEEE In NORCHIP, 2011; 1-4.

[4] Dubois E, and Venetsanopoulos A. A new algorithm for the radix-3 FFT. IEEE Transactions on Acoustics, Speech, and Signal Processing.1978;26(3):222-225.

[5] Fan X. A VLSI-oriented FFT algorithm and its pipelined design .IEEE International Conference on Signal Processing. 2008; 414-417.

[6] Salh A, Audah L, Shah NS, Hamzah SA. Maximizing Energy Efficiency for Consumption Circuit Power in Downlink Massive MIMO Wireless Networks. International Journal of Electrical and Computer Engineering (IJECE). 2017; 7(6).

[7] Raut S N, and Jalnekar R M.Performance Enhancement in SU and MU MIMO-OFDM Technique for Wireless Communication. A Review. International Journal of Electrical and Computer Engineering.2017; 7(5): 2459.

[8] Algnabi Y S, Aldaamee F A, Teymourzadeh R, Othman M, and Islam M S. Novel architecture of pipeline Radix-22 SDF FFT based on digit-slicing technique. IEEE International Conference on Semiconductor Electronics (ICSE). 2012; 470-474.

[9] Suman S, Sharma KG, Ghosh PK. $250 \mathrm{MHz}$ Multiphase Delay Locked Loop for Low Power Applications. International Journal of Electrical and Computer Engineering (IJECE). 2017; 7(6).

[10] Jayakumar D, and Logashanmugamn E.Design and Implementation of Pipelined Radix-2 SDF-SDC FFT Using Modified Carry Select Adder. IIOAB. 2016; 7:137-143.

[11] Naik M V K. Design of carry select adder for low-power and high speed VLSI applications. IEEE International Conference on Electrical, Computer and Communication Technologies (ICECCT). 2015; 1-4. 